tremens, and in whom it was found impossible to bring the bones into apposition or to secure union. Efforts in this direction having failed, her leg was removed below the knee on May 10th, two months after her accident, by lateral Stephen Smith flaps - a method of flap-formation, by the way, which is highly to be commended in amputation at the seat of election. The position of the flaps rendered a drainage tube unnecessary, although a very large amount of oozing was expected from the odematous state of the tissues. The oozing of blood was very great indeed, but in the course of forty-eight hours, at the second dressing, it was seen through the opening which had been left at the most dependent part of the wound that the flaps were filled and distended with a large blood clot. This clot was subsequently watched with much interest. The skin healed by first intention, and at no time was there any pus. The clot gradually contracted in size, and became firmer and firmer, and it clearly aided largely in the filling up and consolidation of the stump, which was perfectly sound and firm when the patient left the hospital on June 16th. The stump had then, in fact, the appearance of one which had been formed for several months, and was absolutely free from discomfort. There can be no doubt, I think, that in this case the blood clot did what Professor Turazza claims for it, and it raises an important question as to the use of the drainage tube. Speaking as the result of general observation and experience, and without reference to any particular case, I should say that the sooner a drainage tube is removed the better is it for the healing process - that is, in aseptic wounds inflicted by the surgeon. The drainage tube performs its office of carrying off superfluous blood and sanguineous serum in the course of from sixteen to twenty-four hours. If its margins have been accurately adapted, the skin wound is healed in this time-sufficiently healed, that is, to render the parts beneath occluded from the air,-and any subsequent extravasation of blood, of which there is sure to be some in the course of the next forty-eight hours, will be dealt with by the tissues, as the tissues ordinarily deal with blood poured out in a true subcutaneous injury where there is no communication with the outside. Some of this blood will be reabsorbed, some-perhaps the greater part-will clot, and organisation forthwith proceeds in this blood coagulum, which fills up all the nooks and crevices of the wound. The clot acts as a sort of scaffold for the building up of new tissue; in other words, organisation takes place on it and in it, and the process of union, of healing, and consolidation is much helped thereby. If the drainage tube be left longer than the time named it is very prone to act as a source of irritation, and I feel sure that it is the frequent cause of suppuration and of apparent failure in antiseptic methods, simply because it has been retained too long. Seeming to act with perfect and needful efficiency because of the visible discharge on the dressings, it is all the while merely carrying off the fluid which itself has caused the tissues to exude. Professor Turazza would discard the tube al together, and encourage the collection of blood and formation of clot, and his opinion clearly deserves the consideration of surgeons. Without $g$ ing as far as he, I would say that each case must be dealt with according to its own conditions, and by no hard-and-fast rule. After some wounds no drainage tube is obviously needed, but in the large majority of cases where it is, the early removal of the tube will, I am convinced, conduce to the healing process and lessen the risks of failure and of suppuration. Accurate apposition of the skin, a dry dressing, and perfect rest-these are the essentials for rapid healing in the presence of ordinary cleanliness, by that obtained by purification of water, or with this or that antiseptic solution. With these precautions, and the very early withdrawal of the tube, the immediate healing of a wound seems, in my experience, to be a matter of almost absolute certainty ; it matters not whether it be the extensive wound in an amputation of the thigh, an arthrectomy of the knee or the excision of a large breast, or the smaller wound such as is inflicted in the operation for strangulated hernia or the removal of a small lipoma. The surgeon secures the immediate closure of the wound, and all inflammatory action which may be set up in deeper parts by the injury of the operation goes on under subcutaneous conditions. We only need to use the drainage tube for the removal of that fluid which, if excessive and causing tension, will interfere with the perfect rest and apposition of the divided tissues. The use of the tube for this purpose is rarely demanded for more than from sizteen to twenty-four hours, and the method of its gradual re moval by cutting it a little shorter day by day is, I think, vastly inferior to that of its bodily removal at the end of the first day, or at the first dressing on the day after the operation, in less than twenty-four hours, even if ito presence beyond this time be not positively injurious to the tissues and does not interfere with the process of healing.

The antisepticism of modern surgery has sometimes been derided, and it has been held that the drainage tube is the real source of all the good which is to be had from antiseptic treatment. The spray has had its day, and it will be strange if the drainage tube is to follow it. Pace Professor Turazza, 1 find it hard to believe that the drainage tube will disappear entirely from use, even in aseptic wounds. Experience, it is more probable, will lead to its being employed with greater discrimination and caution, and with better adaptation of means to ends, than heretofore; and towards this desirable result (for by it we shall have greater certainty in the healing of wounds) the paper of the Italian surgeon on the value of the hæmorrhage in the treatment of wounds cannot be without influence.

\section{NOTES ON ULCERATIVE ENDOCARDITIS. ${ }^{1}$}

\section{BY HENRY MaCLURE, M.D.}

ANy contribution to the literature of ulcerative endocarditis must prove interesting to the profession, and the two cases I am about to give should prove doubly so by reason of the recovery of the patients. The first case is a recent one, the duration of the illness being sixty-eight days-that is, from the beginning till the temperature settled steadily down to the normal.

Miss C-_, aged twenty-six, fairly well nourished, rather anæmic, menstrual function normal. Gouty family history. No history of rheumatic fever or rheumatism. Was seen first on Oct. 9th, 1886. Had complained of some pain in the back and chest for the previous week. She was coiled up in bed in extreme pain, which was referred to the region of the left kidney, with excessive tenderness on pressure. Pulse 80 ; tongue clean; temperature normal; urine cloudy, containing copious lithates, but no albumen. A morphia and ether mixture was given, with chloroform and opium applied externally.

Oct. 10th (second morning).-Still in great pain, which now seems to shoot from the original seat up the back to the left shoulder, with very anxious look; complexion dusky; pulse 109 ; temperature $103^{\circ}$; a distinct direct murmur heard at the second interspaces; some tenderness all over the abdomen; tongue coated with smooth white fur; thirst; no headache or nausea. Evening (7 P.M.): In much the same state; temperature $101.5^{\circ}$; pulse 100 . Ordered salicylate of soda, in ten-grain doses, every two hours.

11th (third day).-Temperature $104^{\circ}$; pulse 110 ; pain less; very restless night; some delirium; urine contained traces of albumen (sp. gr. 1020); systolic murmur heard up into the neck, rasping in character. Evening temperature $101.6^{\circ}$; pulse 120 , and very feeble. Brandy given and salicylate discontinued; quinine in four-grain doses to be given every three hours. Bowels had acted; motions natural in character. An Austin's inhaler with carbolate of iodine is now to be used frequently.

12th (fourth day).-Temperature $102^{\circ}$; pulse 106 . Some delirium in the night; severe headache; no nausea; pupils react to light.

13th (fifth day).-Temperature $105^{\circ}$; pulse 120 ; respiration 28; headache; some delirium. Lungs carefully examined; nothing abnormal. Evening temperature $101^{\circ}$ pulse 110.

14th (sixth day).-Temperature $102^{\circ}$; breathing very shallow; respiration 28 ; constrictive pain round lower par' of chest. Urine and fæces passed involuntarily. Evtning temperature $103^{\circ} ;$ a roseolous rash observed on abdomen.

15th (seventh day).-Morning temperature $101^{\circ}$; some sickness, delirium, and headache; urine albuminous; sp. gr. 1018. As there is great difficulty in using the inhaler, an oro-nasal one was now substituted, with sponge saturated

1 Abstract of a paper read before the Norwich Medico-Chirurgical Society. 
with carbolic acid, creasote, and iodine. There is pain over the heart and distinct cardiac distress.

16th (eighth day).--Temperature $101^{\circ}$; almost constant, rather noisy, delirium in night; breathing shallow ; respiration 30 ; moist râles heard over both bases; pain and tenderness over kidney, spleen, and liver; aortic systolic murmur, harsh and grating, heard all over chest. Seen by Dr. Bateman of Nor wich. Quinine continued; small dose of grey powder; six ounces of brandy in twenty-four hours to be taken.

17th (ninth day).-Temperature $101^{\circ}$; very delirious in night, severe headache; passed evacuations involuntarily; pain, tenderness, and some enlargement of spleen; moist râles, with distinct creaking sounds heard at both bases aortic murmur harsh; other sounds murmurish; pulse tracing dicrotous and feeble; slight jaundice.

19th (eleventh day).-Temperature $1005^{\circ}$; headache less; better night; tongue fairly clean; takes nourishment well. Evening temperature $1028^{\circ}$

23rd (fifteenth day).-A slight rigor had been noticed in the night for the first time. Temperature $104^{\circ}$; pulse 120; respiration 30 ; headache and delirium. Called in the night, as she was thought to be dying. Temperature $1026^{\circ}$ pulse 120, very feeble. (See pulse tracing.) Tincture of digi-

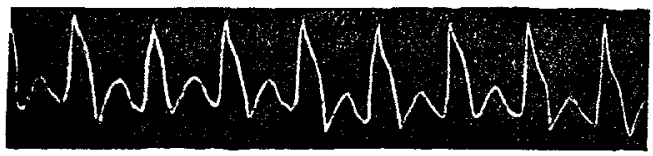

Pressure 21 ounces.

talis in three-minim doses was given every two hours, and brandy increased to eight ounces in twenty-four hours.

24 th (sixteenth day).-Temperature $103^{\circ}$; feels rather better: headache less; pulse tracing better. Evening temperature $101 \cdot 6^{\circ}$

25 th (seventeenth day). - Temperature $100 \cdot 6^{\circ}$; pulse 109 ; respiration 29. Better night. A copious deposit of uric acid sand in urine, with trace of albumen. Cardiac murmur is decidedly softer in character; moist râles and creaking sounds heard over back and sides; a deep inspiration causes severe constrictive pain across lower part of chest; pain and tenderness over left kidney; tenderness over liver and spleen; bowels acting regularly; tongue almost clean takes food well; still some yellow tinging of conjunctivæ.

30 th (twenty-second day). - Slight rigor in night. Temerature $103^{\circ}$; tenderness over heart, liver, spleen, and leftp kidney; still som $\theta$ jaundice; condition of lungs and heart the same. Small doses of solution of perchloride of mercury, with ammonia and cinchona, given.

Nov. 6th (twenty-ninth day).-Temperature $100 \cdot 1^{\circ}$. No headache; no delirium; jaundice almost gone. Condition of heart and lungs the same. A full inspiration still causes pain; tenderness over heart, liver, spleen, and kidney continues. Urine free from albumen; still some uric acid sand.

12th (thirty-fifth duy). - Evening temperature $102 \cdot 4^{\circ}$ pulse 125; tracing of exceedingly low tension; feeling of faintness on sligbtest movement. Brandy increased; tincture of digitalis in five-minim doses, with cinchona given every three hours.

13th (thirty-sixth day).-Pulse rather better; physical signs the same.

21 st (forty-fourth day). - Slight rigor. Temperature 102:50 22nd (forty-fifth day). - The cardiac murmur was observed to be much softer in character.

For several days there was some nausea and sickness, The digitalis was discontinued, and bismuth and soda given. On Dec. 3rd (fifty-sixth day), the morning temperature was $98.8^{\circ}$, and the patient felt decidedly better. Tne lungs were free from abnormal sounds.

9 th (sixty-second day).-Morning temperature $100^{\circ}$. From this time the temperature remained normal till the $16 \mathrm{th}$ when it rose to $100^{\circ}$, and it has since remained normal. (See temperature chart.)

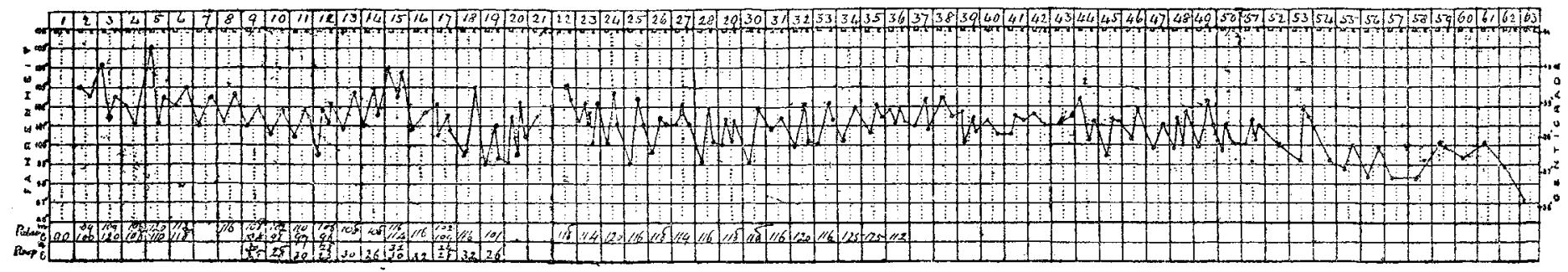

A condition of rather obstinate constipation was the only troublesome after-symptom I had to deal with. There was a steady but slow gain of strength from day to day. She got up for the first time on Jan. 4th. A brownish staining has replaced the roseolous rash. A deep inspiration still causes pain referred to the lower part of the chest in front. There is some slight tenderness over the liver, spleen, and kidney. A soft blowing systolic murmur is still heard propagated along the course of the larger vessels. Other heart sounds normal; still some tenderness on pressure in cardiac region. Has gained flesh; takes food well.

Remarks. - The recognition of ulcerative endocarditis as a distinct disease is comparatively recent. According to Osler $^{2}$ it occurs under three conditions: 1. As a primary disease affecting the lining membrane of the heart or its valves, either attacking persons in previously good health, or more often attacking the debilitated or those with old valve lesions. 2. As an associated condition in connexion with such diseases as rheumatic fever, pneumonia, scarlet fever, diphtheria, ague, \&c. 3. An associated condition in septic processes, traumatic or puerperal. I consider this case a typical one of the disease beginning at the heart. There was no evidence of any previous heart lesion; the fever was secondary to the presumed renal embolism. The main diagnostic points are: 1. The endocardial murmur, its changing character during the illness, and its total disappearance. 2. The character of the fever as indicated by the thermometer, its irregularity of type, the exclusion of other affections-as rheumatic fever, pneumonia, \&c. 3. The albuminuria, roseolous rash, jaundice, and the presumptive evidence of infarcts in kidney, spleen, and lungs. There was no initial rigor observed, and only three slight ones during the illness; there was no cough till the last week, and that

2 THE LAXCET, vol. i.., 188. was transitory in character. There was no irregularity of pulse observed, and no hæmaturia.

The disease is considered to be due to the presence of 8 micro-organism - the staphylococcus. These microbes exist in colonies on the endocardium, are swept into the blood current, lighting up a septic fever, and are found in the plugs of the vessels of the spleen and kidney. From what I have gathered from the literature of the subject, the prognosis is gloomy in the extreme. Almost all the recent authorities state that there has not yet been one recorded case of recovery, but admitting the anatomical possibility of it.

As to the treatment. Believing the disease due to the presence of a micro-organism, and a micro-organism of low vitality, the germinating power of whose spores is completely checked by a solution of 1 in 300,000 of bichloride of mercury, a rational method of treatment would seem to be to expose the blood to the action of an antiseptic vapour. which we can do by means of inhalations. This method of treatment was suggested to me by the following case.

June 2nd, 1882,-J. B- aged twenty, first seen on this date, had been ill for about ten days under Dr. Stovin-an undefined illness : some dysenteric symptoms, with obscure pains in muscles and joints; slight fever; was able to walk about his room.

3rd.-Confined to bed. Temperature $102^{\circ}$. Some cough, with difficulty in breathing; a well-marked mitral systolic murmur heard at apex for the first time. For a few days in much the same state. There was a good deal of nausea and sickness.

About June 8th a well-marked phlegmasia alba dolens developed in the left leg. Cough much worse; some dulness at base of left lung; extreme fetor of bresth, with profuse expectoration of extremely fetid greenish matter. We then determined to try antiseptic inhalations. An Austin's 
inhaler was used with carbolate of iodine. In a very few days a marked improvement set in, the swelling of the leg was almost gone, the lung symptoms were decidedly better, and the fetor less; some broken down lung tissue was expectorated, the murmur at the apex still persisting. He went on steadily improving, and in about fourteen days was almost well. There was still some cough and expectoration, but no fetor. The cardiac murmur was softer in character. Before the end of August he was able to get out; cough gone, and the heart-sounds quite normal. I saw him a year after a perfect picture of health. The lung sounds were perfect, and the heart quite normal.

That this was a case of ulcerative endocarditis I have no doubt now, but at that time there was not much known on the subject by the ordinary general practitioner. Both Dr. Stovin and myself attributed his recovery to the inhalations, and, I think, rightly so, as amendment in all the symptoms began almost directly after using the inhaler.

In the December following this, Dr. Julius Pollock related a case of ulcerative endocarditis with pyæmic symptoms, ${ }^{3} \mathrm{re}-$ sulting in death on the fifteenth day. He says: "The treatment of ulcerative endocarditis with pyæmia is most unsatisfactory; none of the ordinary remedies had any real effect, nor did large doses of alcohol sensibly improve matters. I wish now I had tried the inhalation of carbolic acid or carbolate of iodine, and I would suggest giving such a line of treatment a fair trial in any case of the kind, especially where the lungs appear to be much affected." The case, I may say, was watched attentively throughout by Dr. Stovin (now of Stratford) and myself. I am sorry my notes are defective, but $I$ think the main points and course of the case are correctly indicated. I have recently submitted them to Dr. Stovin for his verification.

In my recent case, though the inhalations were begun on the third day, I am afraid they were practically useless until the seventh day, when the oro-nasal inhaler was substituted. This was used throughout the illness for ten minutes at a time, about ten or twelve times in the twentyfour hours. I cannot help feeling that the gradual decline of the most urgent symptoms was due to the presence of the antiseptic in the blood, checking the growth and reproduction of the microbes, if not destroying them. The second indication in the treatment of my recent case was to keep up the failing power, and especially to combat the cardiac depression. Fortunately, nourishment was taken fairly well throughout (administered often), and consisted of strong broths, beef-tea, jellies, milk, eggs, \&c. Stimulants were givenfreely when absolutely required. Digitalis, though often indicated, was given with caution, fearing to excite sickness or nausea ; the sphygmograph was specially useful in giving the indication for it and recording its effects. During the most anxious part of the illness I was fortunate in having the co-operation and assistance of the young lady's brother, a very intelligent senior medical student, who watched the case most carefully, and to whom I am indebted for the minute records of the temperature. As he often heard the cardiac murmur curing the illness, he tells me he auscultated the heart recently, and that the sounds seemed to him quite right; he could detect no murmur. This I can verify, as I saw my patient on March 26th; she was then able to walk a mile without fatigue. The heart sounds were normal and the lungs sound.

\section{CASE OF ACUTE RHEUMATISM WITH HYPERPYREXIA.}

\section{BY ARTHUR J. DALTON, L.R.C.P. LOND., M.R.C.S.}

I READ with much interest Dr. Taylor's case of rheumatic hyperpyrexia in THE LANCET; and having had a very similar one lately in private, with even a higher temperature-viz., $108 \cdot 6^{\circ}$ in the axilla,-I venture to publish a few particulars of it.

Miss S-, aged twenty-four, never had rheumatism before, but her father, mother, and one sister had suffered from it on several occasions. On Dec. 31st the patient was seized with pains in the joints, which shortly became swollen and hot. On the fourth day she had a little pleurisy on the left side, with a good deal of pain. The temperature was never rery high. On January 6 th the pain in the joints disappeared, and an erythematous rash appeared over the body. The temperature now began to rise, and on the morning of Jan. 7 th was $103^{\circ}$. Up to this date I had only seen the patient once, my partner, Mr. F. M. Miller, having been in attendance. In the afternoon of the same day, at 3.50 P.M., they sent round in a great hurry, and, Mr. Miller being out, I went at once, to find the patient violently delirious, erythematous rash covering the body, pulse very feeble and not to be counted, and the temperature in the axilla $108 \cdot 6^{\circ}$. I immediateiy ordered half an ounce of brandy with a little milk, which was given by the mouth with great difficulty. The patient was then stripped, and a sheet wrung out with ice-cold water applied to the chest and abdomen; also several lumps of ice, four or five in number, were placed outside the sheet. The patient was so violently delirious that it took three people to hold the ice on and keep her in bed. The sheet was replaced by another at short intervals and fresh ice applied, - 4.5 P.M.: The brandy and milk, with a quantity of milk previously taken, was vomited, also a good deal of phlegm. 4.15 P.M.: Temperature $107^{\circ} 6^{\circ}$; pulse $160 .-4.45$ P.M.: Temperature $104^{\circ}$ pulse 124. Patient passing everything under her, and motions very much relaxed and offensive. -5 P.M.: Temperature $1024^{\circ}$; pulse 124 , weak. Patient now fairly conscious, but much hesitancy in speech and difficulty in finding words; good deal of muttering and trembling of lips. Subcutaneous injection of brandy (ten minims).5.20 P.M. : Temperature $1006^{\circ}$; pulse 128, stronger. Injection of brandy (fifteen minims) subcutaneously. -5.40 P.M.: Temperature $99^{\circ} 6^{\circ}$; pulse weak. The ice was now removed, patient dabbed dry, and put into another bed in the same room between blankets. She was now quiet and conscious.6 P.M.: Temperature $97 \cdot 2^{\circ}$. Injection of brandy (fifteen minims) repeated, and, as patient complained of feeling cold, a hot bottle was applied to the feet.--9.30 P.M.: Temperature $99^{\circ}$. Quite conscious and comfortable.-12 midnight: Temperature $102^{\circ}$; pulse good. Face a little flushed. Ice was applied to the head, and ten grains of sulphate of quinine were given in a little orange juice. The patient was given small quantities of brandy and milk during the night. The temperature taken through the night by patient's sister was as follows: at 1 A.M., $102^{\circ}$; 2 A.M., $103^{\circ}$; 3 A.M., $103^{\circ}$; 4 A.M., $102^{\circ}$; 5 A.M., $103^{\circ}$; 6 A.M., $103^{\circ}$; 7 A.M., $104^{\circ}$. The patient was also given five grains of sulphate of quinine every two hours.

On seeing the patient at 8 A.M. on January 8th, I found she had taken a pint of milk during the night, and about seven dessertspoonfuls of brandy. There had been no sickness. Her temperature was $1033^{\circ}$, and pulse 120 , full and regular; respiration 36 . The patient complained of feeling tired, and seemed inclined to sleep. She had had a fair amount of sleep during the night, and had passed a large quantity of urine naturally, having passed nothing more under her.-11.40 A.M. : Temperature $1018^{\circ}$; pulse 109 ; respiration 33 . Since 8 A.M. has had six dessertspoonfuls of brandy with milk and quinine, five grains every two hours. She is now a little deaf, with some buzzing in ears, and very drowsy. No headache. Last quinine powder was vomited. The brandy and quinine to be discontinued.4 P.M.: Temperature $101 \cdot 6^{\circ}$; pulse 110 ; respiration 37 . The breathing resembles Stokes' respiration. Since 12 o'clock has had no brandy, but has taken a pint of milk without sickness. Quinine (five grains) to be repeated. Ice still to head. The patient expresses herself as much better. The bowels have acted well; motions pale, but not relaxed.9.15 P.M.: Temperature $1015^{\circ}$; pulse 100 ; respiration 36. Has slept a good deal during the day. Takes plenty of milk. The quinine now to be given every four hours. The temperature during the night did not exceed $101.4^{\circ}$.

Jan. 9th.-10.30 A.M.: Temperature $100^{\circ} 8^{\circ}$; pulse 93; respiration 32, more regular and less noisy. Quinine given every six hours. No pain in any of the joints,-9.20 P.M.: Temperature $1004^{\circ}$; pulse 92 ; respiration 32, quiet. This evening the patient says voices have been talking to her. Head has been a little uncomfortable. Since 5 P.M. has had a little pain in the left knee and ankle, also in the wrists, but there is no swelling or redness. Quinine to be discontinued, also ice to be removed from head. Both to be repeated if the temperature reaches $103^{\circ}$

10th.-Breathing normal. No pain anywhere. Temperature through the day varying from $99^{\circ}$ to $100^{\circ}$. The skin has resumed its normal appearance.

11th. - Temperature throughout the day $99^{\circ}$.

12th.-About 11 o'clock last night the pain in the wrists F 2 